\title{
Modelling the behaviour of engine assembly workers
}

\section{Tim Baines and John Ladbrook}

\section{Introduction}

Once, direct workers were considered to be a necessary evil, soon to be replaced by robotics and automation. Today, many manufacturers appreciate that people in these roles can provide important flexibility and responsiveness. This recognition has been demonstrated by the many newer initiatives that emphasise developing the capabilities of the individual rather than attempting to remove them from the production system.

It is a principal responsibility of the Engineers and Managers within an organisation to create the working environment that helps to deliver the best performance from their people. Here, the term environment does not just refer to physical factors such as temperature and noise, but means the whole social, economic and physical conditions of the workplace. For example, the extent of available interaction with colleagues is an environmental condition, so too are the pay and reward schemes. In this paper we present a prototype modelling methodology that enables the creation of quantitative models of the relationships between working environment, the direct workers and their subsequent performance. Once created for an organisation, such models can provide prediction on how the behaviour of their workers will alter in response to changes in their working environment. The goal of this work is to improve the decision processes used in the design of the working environment. Our intention is that through improving such processes, companies will gain better performance from their direct workers, and so improve business competitiveness.

This paper is structured to first present the need to model the behaviour of direct workers in manufacturing environments. To begin to address this need, a simplistic modelling framework is first developed, and then expanded to provide a detailed modelling methodology. There then follows a description of an industrial evaluation of this methodology at Ford Motor Company.

\section{A modelling framework}

If a means could be found to provide models of the relationships that exist between an environment, the workers and their performance, then this would be a valuable tool in the design of manufacturing organisations. Practitioners could use this approach to model their own organisations, and then use the model to improve their understanding and predictions about the effect of changes to the working environment on the people within their plant.

The motivation that an Engineer or Manager would have for modelling, is that it would lead to improved manufacturing competitiveness. Modelling can help to identify strategies to improve the working environment, or help to select the best people to work in a particular environment. A robust model could be used independently to give localised indications of performance. Alternatively, such a model could also be combined with other forms of manufacturing system model such as DES, to give an holistic view of business performance (Baines et al, 1998).

To create such a model the user would need to adopt a procedure of data collection, validation and experimentation. The modelling process would begin by collecting data about the existing system and building this into a basic model. This model would then be validated, by ensuring that its behaviour accurately represented the performance of the real systems. Once validated, the model could be experimented with, and its outputs taken to be strong indications about the affects of changes to the real systems.

Some simple modelling approaches do exist. As early as Lewin (1935), a conceptual modelling structure was encapsulated by the expression:

$$
B=f(P . E)
$$


Where:

$\mathrm{B}=$ Modes of behaviour, $\mathrm{P}=$ Personality trait or whole personality, $\mathrm{E}=$ Somatic and psychological environment of the individual.

To reflect the motivation of Engineers and Managers, the principal output from a model needs to be performance measurements that indicate how worker behaviour will be affected by an environmental change. This relates to the symbol ' $\mathrm{B}$ ' in the Lewin (1935) expression given above. Furthermore, the principal input into a model needs to be information that describes the people and their environment, as these are the factors with which Engineers and Managers would want to experiment. This relates to the symbols ' $P$ ' and ' $E$ ' in the Lewin expression. Finally, the model itself needs to capture the relationship between input and output, relating to ' $f$ ' in the Lewin expression. Collectively, the inputs, outputs and relationship model represents a simple view of the required modelling capability.

Our modelling methodology is based on the principles of modelling established by Lewin (1935). A schematic illustration of the methodology is given in figure 1. This methodology was developed to model the relationships between direct workers, their working environment, and their subsequent performance. In short, this pilot methodology roughly captured the relationships between environmental conditions, the personality profile of direct workers, and their performance. This relationship was then represented as a computer based model that used an Artificial Neural Network to provide predictive capabilities. This network was populated by gathering data about the behaviour of 125 assembly workers at other plants. The validity of this tool was then demonstrated in tests with Ford. A brief explanation of how this is applied given below:

Step 1. Define outputs required from a model. Describe the behaviour of a direct worker in terms of core production tasks. For each core task, identify relevant numerical performance indicators. For example a core task could be product assembly, for which indications would be given about how the time taken might vary.

Step 2. Define the experimental variables as inputs to a model. Two sets of variables are used, the first are measures of the working conditions under consideration, such as temperature, light, noise. The second are the personality profiles of the people who carryout the core tasks, and this is carried out using a Briggs Myers personality test.

Step 3. Collect relationship data and train the Artificial Neural Network (ANN). Study similar production tasks in other working environments, using a formalised method of data collection to capture the relationship between environment, person and performance. Training then takes place by stimulating the ANN with a set of inputs for which the outputs are known, and then teaching the ANN how to respond.

Step 4. Experimentation with the validated model. Once validated, the model can used by the practitioner to explore, within the scope of the model, how the design of the working environment can affect the performance of direct workers.

\section{Application of the methodology: a case study in engine assembly}

The modelling methodology is a concept, it is simply an approach to building models that capture the relationship between people, their environment and behaviour. The purpose of such models is to improve the decision making within manufacturing companies about the design of the working environment. This section describes work that has been carried out to determine whether the proposed methodology does indeed fulfil the intended purpose.

The methodology is embryonic, therefore the evaluation described in this paper only set out to assess the validity of the principles of which the methodology consists. The intention was to simply consider whether the methodology 'can work', rather than conduct an exhaustive critique to see 'how well' it works in a variety of applications. Ford Motor Company has been a long-term sponsor of modelling research at Cranfield, and they offered to participate in the evaluation work. A UK assembly plant was visited, and one engine assembly department was chosen as a 
suitable modelling case. The challenge was then to build a model of this department, which would give valid predictions of how the performance of the assembly workers would alter if the working conditions changed.

To operationalise the modelling methodology in this initial study, a basic computer tool was created using Visual Basic software. Visual Basic was chosen due to its flexibility, ease of the implementation of mathematical functions, and feasibility of linking with databases and other software. The subsequent modelling tool was structured around the modelling methodology, and incorporated within it an ANN framework. Once complete, model building could commence.

In a typical engine assembly line, many work-stations exist where a wide variety of components are assembled onto an engine by direct workers. The first step in model building was therefore to establish a set of core tasks that represent the activities at these workstations. The Cranfield researchers carried out such rationalisation and found seven different core tasks for engine assembly at this Ford plant, namely: pick and place, fastening, adjusting, stock handling, release job, quality assurance and inspection. To complement the core tasks, three basic performance indicators were then identified, and these were; cycle time, rejection rate, and parts waiting for labour.

The second step in model building was to establish the personality profiles of the workers in the department being considered. A questionnaire was constructed that asked workers about themselves, their tasks and their environment. This questionnaire was based on Briggs Myers (1980) style questions that had been tailored to suit the context of this study. This questionnaire was completed by the researchers during semi-structured interviews with twenty workers in the department. Subsequent analysis then revealed that the personalities of these workers fell naturally into one of four different stereotypes.

The third step in model building was to identify the environmental conditions around which to configure the model. These conditions needed to reflect the environmental changes with which the Ford Engineers wanted to experiment. Through discussions, sixteen environmental states were chosen and these included; noise level, ventilation, temperature, daylight, and cleanliness. These environmental factors were mainly physical as the researchers found these most easy to describe and capture.

The fourth step in model building was to capture data that described the relationship between environment, personality and behaviour in this case. Again, a questionnaire was formed, and applied using semi-structured interviews. This questionnaire used a series of carefully poised questions that asked the interviewee how they would respond to a variety of changes to their working environment. The questions were set so that responses could be cross referenced, and so checked for inconsistencies, which if found were removed from the data. The questionnaire also incorporated questions about personality, so that responses to environmental conditions could associated with personality stereotype. This time, the interviewees were assembly workers at Ford and other assembly plants in the UK. Ten manufacturing plants were visited, and this provided data from 125 assembly workers. All this data was then used to populate the ANN model.

Evaluation of the modelling methodology was based on testing to see whether the model that had been created could provide reasonable predictions about worker behaviour. The test was sympathetic to the limitations of the data and the possibility of bugs in the newly created modelling software.

The chosen test therefore, was to invite a small group of Ford Engineers to sit with one member of the Cranfield research team. These Engineers were chosen because they held expert knowledge about the people and the department being modelled. The purpose of the modelling tool was explained to the group, and then they were asked to suggest a number of tests for the tool. A series of changes in the environmental conditions were then determined, and one by one input into the model. For each change, the model provided information about the performance of workers against each of the generic tasks. The Ford Engineers were asked to score the results. A high positive score was awarded for a very accurate prediction, whereas a high but negative score was awarded for an inaccurate prediction. 
The model did not provide perfect performance. On the very first attempt, an environmental condition was chosen for which a wildly inaccurate response occurred. On investigation, it was apparent that this inaccuracy occurred because the ANN had insufficient data on which to form a valid response. However, once the questions from the Engineers corresponded to the available data, then valid responses were given by the ANN. Where credible data existed, the model performed acceptably. On completion of the evaluation trial, the responses of the Ford Engineers supported the validity of the model. This was purposely an un-ambitious trial, however it can be concluded that it has demonstrated that the modelling methodology is valid.

\section{Conclusions}

This paper has presented a modelling methodology that is intended to provide a generic approach to modelling the relationship between direct workers, their environment and their subsequent performance. This methodology has been assessed in one study and in this =se has been found to be valid. There are many challenges that this theme of research needs to address. The work described in this paper has made an important first step in this area, having gone someway to establishing a generic methodology and illustrating the potential value. Our future work will now build from this foundation.

\section{References}

Baines, T.S., Harrison, D.K., Kay, J.M., and Hamblin, D.J., 1998, A consideration of modelling techniques that can be used to evaluate manufacturing strategies, Int. J. Adv Manufacturing Technology, 14:369-375.

Lewin, K., 1935, A dynamic theory of personality, McGraw-Hill.

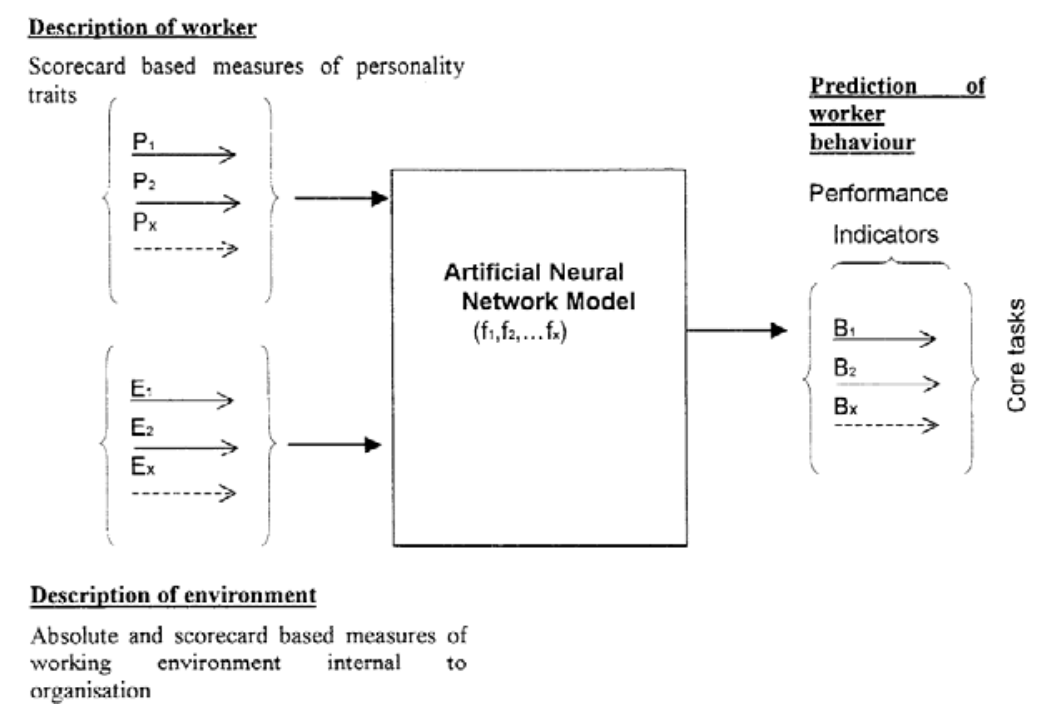

Figure 1: The modelling methodology 